\begin{tabular}{|c|l|}
\hline Title & Effects of Fluid Directions on Heat Exchange in Thermoelectric Generators \\
\hline Author(s) & Suzuki, Ryosuke O.; Sasaki, Y uto; Fujisaka, Takey uki; Chen, Min \\
\hline Citation & $\begin{array}{l}\text { Journal of Electronic Materials, 41/6), 1766-1770 } \\
\text { https:/doi.org/40.1007/s11664_012-2074-x }\end{array}$ \\
\hline Issue Date & 2012-06 \\
\hline Doc URL & http://hdl.handle.net/2115/49418 \\
\hline Rights & The final publication is available at www.springerlink.com \\
\hline Type & article (author version) \\
\hline File Information & JEM41-6_1766-1770.pdf \\
\hline
\end{tabular}

Instructions for use 


\title{
Effects of Fluid Directions on Heat Exchange in Thermoelectric Generators
}

\author{
Ryosuke O. Suzuki*,**, Yuto Sasaki, Takeyuki Fujisaka, and Min Chen*** \\ Faculty of Engineering, Hokkaido University, Sapporo 060-8628 Japan \\ * Contributing author, rsuzuki@eng.hokudai.ac.jp \\ Tel: +81 11706 6339, FAX: +81117066342 \\ ** JST-CREST researcher \\ *** JSPS Post-doctoral fellow, on leave from Aalborg University, Denmark
}

\begin{abstract}
Thermal fluids can transport the heat to a wide surface of the thermoelectric (TE) panel from the hot and/or cold sources. The obtainable TE power was precisely evaluated using the numerical calculation based on the fluid dynamics and heat transfer. The commercial software FLUENT was coupled with the TE model for this purpose.

The fluid velocity distribution and the temperature profiles both in the fluids and TE modules were calculated in the two-dimensional space. The electromotive force was then evaluated for the counter flow and the split flow models to show the effect of stagnation point. The friction along the fluid surface in a long flat path was larger than that in the short path split into two parts. The power circulating the fluids into the flow path is not so small to be neglected, and this pumping power should be considered to design the TE generation system.
\end{abstract}

\section{Key words}

Thermal fluid, TE power generation, numerical simulation, TE panel 


\section{Introduction}

Thermal fluids can transport the thermal energy to a wide surface of the thermoelectric (TE) panel from the hot and/or cold sources. When the amount of waste thermal energy is limited, the smaller temperature difference between the hot and cool surfaces can not be avoided due to the heat exchange through TE modules. The usage of thermal fluids to give the wider surface area of TE modules is very effective to rise the recovery yield of waste heat[1-7]. However, too large TE panel increases the internal electrical resistance and the TE power drops. Therefore, an adequate dimension of TE power generation system is proposed from the analytical calculation[2-7]. For example, the counter flow of two fluids was reported as the most effective way, in order to obtain the largest TE power from a single flat TE panel[2].

Recently the authors reported from the one-dimensional analysis that the split flow perpendicular to the TE panel can give the same power as the counter flow[7]. However, the fluid behaviors were approximated as uniform turbulent flows and the one-dimensional analysis did not simulate the split effects of fluid correctly. These assumptions cause some errors to describe the heat exchange in the concerned models, if they are applied to the practical power generation.

The purpose of this study is to reports the results of numerical calculation where we can consider the two-dimensional motion of both fluid and heat in the two working fluids, and where we can analyze the two-dimensional temperature distribution both in the fluid and solid simultaneously. The reported fluid pattern such as the counter and split flow [7] is again studied from the two dimensional analysis. The efficiency of counter flow is analyzed using the heat transfer and fluid dynamics in the single TE panel. Especially the effects of stagnation and convection of the fluids are estimated. The commercial software FLUENT was coupled with the TE model for this purpose [8-10].

\section{Modeling for numerical calculation}

A simple and fundamental module was constructed in the grid. П type p-n pair consisting of 4 pieces ( $5 \times 5 \times 5 \mathrm{~mm}$ bricks) is connected in series with the metallic electrode $(5 \times 12 \times 0.025 \mathrm{~mm})$ and ceramic insulator $(0.025 \mathrm{~mm}$ thick $)$. As the 
thermal properties of TE materials, those of the BiTe pair were taken because they were commonly used in the previous studies [2-7]. As the fluid, pure water was chosen and flowed along the surface of TE module. Because the analytical simulation [2,3,5-7] could not handle the inhomogeneous velocity distribution, this paper focuses the velocity and temperature distribution. The fluid dynamic software FLUENT calculates the mass reservation and the momentum reservation simultaneously based on the finite element model. The heat transfer is evaluated by adding the term of energy reservation in these two reservation laws. The typical temperature dependencies for these materials were set from the handbooks. The mesh is set as 10,000 for TE materials, 240 for electrodes and insulator, 20,800 for fluids. FLUENT can handle the velocity and temperature in the both fluid and solid mesh. The flow at the inlet is set homogeneous but soon becomes a parabolic profile of velocity due to laminar flow assumption. The iteration in numerical calculation was converged at 1,000 , because the error for loop calculation becomes much smaller when the iteration is over 300, and because the evaluated result of iteration $=44,500$ was identical with that of 1,000.

Thermoelectric motive force (EMF) as the open circuit voltage was calculated by the add-in program as TE evaluator [8,9]. It calculated firstly under a constant temperature gradient without any fluids, and the effectiveness and reproducibility of calculation was confirmed by varying the calculation parameters such as mesh size. When the mesh size was not adequate, the edge effect of the electrode was significant, which was found in the electric potential analysis. The details will be reported separately. The chosen numerical parameters seem good to express the effect of fluids.

The fluid velocity distribution and the temperature profiles in the fluids and TE modules were calculated in two-dimensional space by setting two thermal fluids. EMF was evaluated for the counter flow and the split flow models to show the effect of stagnation point, which we can not evaluate in the previous analytical approach [7]. 


\section{Results and Discussion}

\subsection{Simple Counter Flow in One Dimensional Array}

\section{(a) Temperature distribution}

The same amounts of water $(298 \mathrm{~K}$ and $348 \mathrm{~K}$ at the inlet) per time were supplied to both two fluids paths, as shown in Fig. 1. The adiabatic walls were set at the top of hot fluid and at the bottom of cold fluid, and the heat will transfer from the hotter fluid (upper path in Fig.1) to the cold fluid (lower path) through the electrodes and TE modules. As the gap for the fluid path was set $1 \mathrm{~mm}$, we limited the maximum velocity by $0.05 \mathrm{~m} / \mathrm{s}$ to confirm the laminar flow. Because the counter flow is assumed in Fig. 1, it can be seen that the hotter fluid (red part of upper fluid) expends the hot zone to the right part as the fluid velocities increase. Oppositely the colder fluid (deep blue part of lower fluid) expands the cool part to the left part. At the higher velocities the temperature distribution in TE elements became flatter, and the similar profiles was found for all the elements as seen in Fig. 1.

Fig. 2 shows the temperature profiles at the inner surface of the electrode, i.e., the surface temperature at the terminals of TE element. It is clear that the temperature of hot fluid decreases along the path, and that the cold fluid is warmed up. The surface temperature of electrode was found continuous even at the position that TE element does not contact to the electrode. Therefore, we may assume that the gaps between the TE elements are not critical to evaluate the temperature distribution along the path.

Although the fluid velocity is set slow, the heat transfer through the solid TE module is much slower. At the higher velocities, most of heat was carried away by the hot fluid without giving any heat to the module. This condition gives the larger temperature difference to the TE module, although the thermal efficiency of the fluid became the worse.

\section{(b) EMF}

The temperature profiles in the TE elements enable us to evaluate the electric motive force (EMF) due to Seebeck effect. This calculation assumes the electric connection with the external load of $0.1 \mathrm{ohm}$. Therefore the current generated is 
so small that the contribution of Pertier cooling can be neglected. However, Fig. 3 shows the EMF evaluated by considering the Pertier effect and temperature dependency of thermal properties of TE elements. The effects of thermal properties of materials on EMF values were not significant as they will be reported separately.

As shown in Fig. 3, the velocity of thermal fluid significantly affects the EMF value. A small increase of velocity can generate the larger TE power. For example, the calculation at $100 \mathrm{~m} / \mathrm{s}$ (extremely fast to correspond to the ideal heating and cooling) gave $19.17 \mathrm{mV}$. When we can set the TE terminals at $298 \mathrm{~K}$ and $348 \mathrm{~K}$ ideally, EMF should be $22.6 \mathrm{mV}$ at the given conditions in this study. This difference $(11.7 \%)$ is due to Pertier cooling and Joules' heat, which were neglected in the analytical calculation $[2,3,5,6]$. The possible temperature drop at the electrodes was not severe in EMF values. This is partially because this model used the metallic silver as the very thin conductor.

Fig. 3 also shows the EMF when the thermal fluids are flown in parallel. The analytical evaluation after simplification predicted that the EMF value in counter flow is always larger than that in parallel flow[2], however, this evaluation shows that it is true only at the slower velocities. Although the difference is very small, the EMF in parallel flow became a little larger than that in counter flow. All the variables in the numerical calculation such as the temperature dependencies of TE elements, fluids, electrode materials and heat transfer coefficients may relate each other in the complex way, and the details will be reported separately. However, this kind of unexpected behavior from the analytical calculation may require the modification of the system design in TE power generation. Thus the importance of numerical calculation was demonstrated.

\subsection{Splitting and Joining Fluids}

\section{(a) Temperature distribution}

The previous analysis by some of the authors [7] showed that the simple counter flow over one dimensional TE array can generate the maximum power when two fluids are used. The layered packing of TE modules could form the similar output, but a slightly smaller output was predicted. When we use the one 
dimensional array of TE modules, the splitting and joining fluids can generate the identical maximum output [7].

As clearly shown in Fig. 4(a), a hot fluid is introduced at the center of TE module and blown to the upper path perpendicularly. It is split into two fluids and flows out at the two terminals of the upper path. In contrast, the cold fluid is introduced from the two ends of the lower path, it joins together at the center of the lower path and extracted from the meeting position. Therefore, the central part of upper path is heated and two terminals of lower path are cooled, as seen in Fig.4(a). When the velocity increases, the temperature profiles becomes homogeneous as seen in Fig.4(b).

The temperature distribution at the adiabatic wall surface corresponds to the representative fluid temperature. As shown in Fig. 5, this strange flow type of two fluids gives the central symmetric temperature profile.

\section{(b) Velocity of Fluid}

The velocity profiles were also analyzed. Although the homogeneous velocity was set at the inlet in any cases, the parabolic velocity profiles were rapidly grown even at the part closer to the inlet. According to the non-dimensional analysis of fluid dynamics, the influence of inlet should exist even at the outlet in these settings, but it seems to be well suppressed.

Fig. 6 shows the velocity analysis near the outlet of the cold fluid in case of "joining fluid". The velocity at the central part of the fluid path is the fastest in the laminar flow, and the direction of fluid changes to a perpendicular direction at the outlet. As the results, the cold fluid path is stagnated at the portion close to the electrode wall, and the fluid stops its motion in x-direction. The detailed analysis of $\mathrm{x}$ - and $\mathrm{y}$ - directional velocity shows that the circulation or rotation of fluid occurs near the stagnation point. In the same way the circulation of hot fluid is generated at the inlet of the fluid. The velocity change was not considered in the homogeneous turbulent flow in the previous studies [2,3,5-7], and this study clearly shows the importance of fluid analysis. 


\section{(c) $E M F$}

The EMF values for split-joining flow are also shown in Fig.3. They are commonly smaller than those in counter flow by about $1 \%$ when compared at the same velocity. The difference is small but it may be due to the existence of stagnation points.

\section{(d) Friction}

Because the non-slip wall was assumed, the friction at the electrode's surface is large, and the larger pressure is needed to drive the fluid. The pressure in the fluid decreased as the x-position approached to the outlet where the pressure was set as 0 . Pressure loss is the smallest at the center of fluid path, and it is as large as $0.031 \mathrm{~Pa}$ at $0.001 \mathrm{~m} / \mathrm{s}$, and $16.2 \mathrm{~Pa}$ at $0.050 \mathrm{~m} / \mathrm{s}$. The proportionality of pressure loss and velocity was known in the fluid dynamics. The friction along the fluid surface for a long flat path was much larger than that for the shorter paths split into two fluids.

In future we can calculate the total pressure loss for the fluid motion, and will evaluate the energy balance between the output from TE power generation and the energy consumption for fluids circulation mechanics.

\section{Conclusions}

The heat transfer from two thermal fluids to TE module was numerically analyzed using the fluid dynamics and heat transfer. Because of the narrow and short path, the laminar flow is assumed with the slow fluid velocity. It is partially because this effect could not be analyzed by the previous analytical approaches.

The electromotive force (EMF) was evaluated for the counter flow and for the split-joining flow models. At the latter model, the effect of stagnation point was found. The friction along the fluid surface was calculated. The pumping power should be considered to design the TE generation system in future.

This paper predicts a modification of TE generator by introducing the fluid separation and its direction change. This model was numerically examined by fluid dynamics and heat transfer, and it was favorable in order to decrease the 
pressure loss of fluids. The idea to use the counter flow should be confirmed experimentally to strengthen the future design of TE generator.

\section{Acknowledgements}

The authors thank the continuous discussions with Prof. K. Koumoto, Dr. R. Funahashi, and Prof. H. Anno. This work was financially supported by JST-CREST project research.

\section{References}

1. K. Ono and R. O. Suzuki, J. Met., 50 (1998) 49-51.

2. R.O.Suzuki and T. Tanaka, J. Power Sources, 122 (2003) 201-209.

3. R.O.Suzuki and T. Tanaka, J. Power Sources, 124 (2003) 293-298.

4. T. Kyono, R.O. Suzuki, and K. Ono, IEEE Trans. on Energy Convers. 18 (2003) 330-334.

5. R.O.Suzuki and T. Tanaka, J. Power Sources, 132 (2004) 266-274.

6. R.O.Suzuki, J. Power Sources, 133 (2004) 277-385.

7. Y. Sasaki and R.O.Suzuki, J.Elect.Mater., submitted.

8. M. Chen, L.A. Rosendahl and T.J. Condra, Intern. J. Heat Mass Transfer, 54 (2011) 345-355.

9. M.Chen, S.J.Anderson, L.A. Rosendahl, S.K.Kaer, T.J. Condra, J.Elect. Mater., 39 (2010) 15931600 . 


\section{Figure Captions}

Fig.1 Temperature distributions of simple counter flow when the fluid velocity is $0.001 \mathrm{~m} / \mathrm{s}(\mathrm{a}), 0.005 \mathrm{~m} / \mathrm{s}(\mathrm{b}), 0.01 \mathrm{~m} / \mathrm{s}(\mathrm{c})$ and $0.05 \mathrm{~m} / \mathrm{s}(\mathrm{d})$.

Fig. 2 Temperature distribution of simple counter flow along the flow direction.

Fig.3 Relationship between Seebeck EMF and fluid velocity of simple counter flow.

Fig.4 Temperature distributions of splitting and joining flow when the fluid velocity is $0.001 \mathrm{~m} / \mathrm{s}(\mathrm{a})$ and $0.05 \mathrm{~m} / \mathrm{s}(\mathrm{b})$.

Fig.5 Temperature distribution of splitting and joining flow along the flow direction.

Fig. 6 Velocity of cold fluid when "joining" occurs at the center. 
(a)
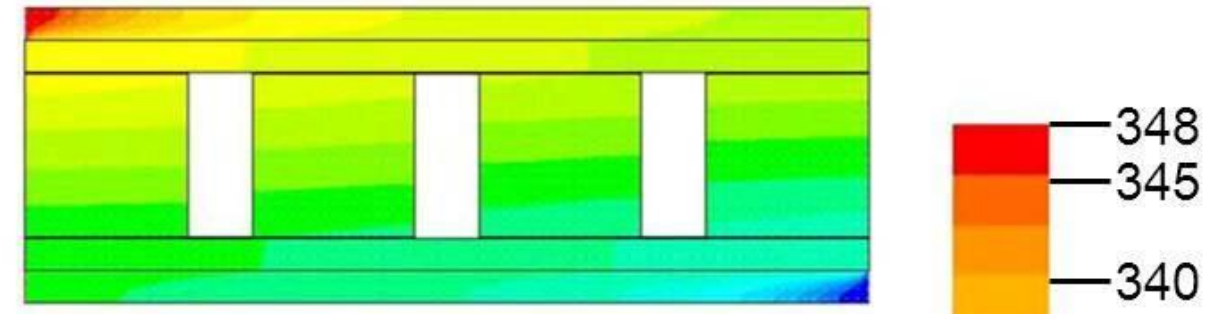

(b)
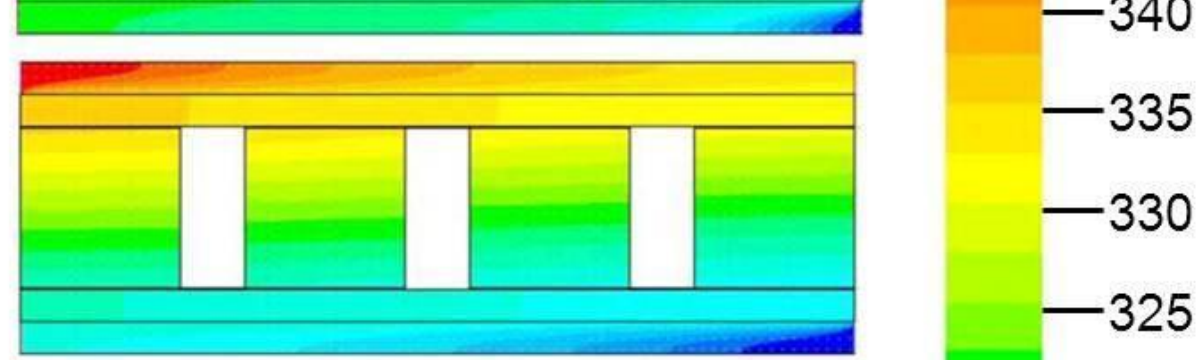

$-330$

$-325$

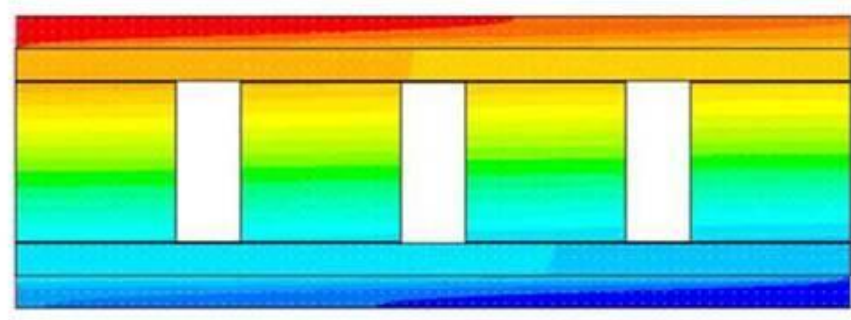

(c)

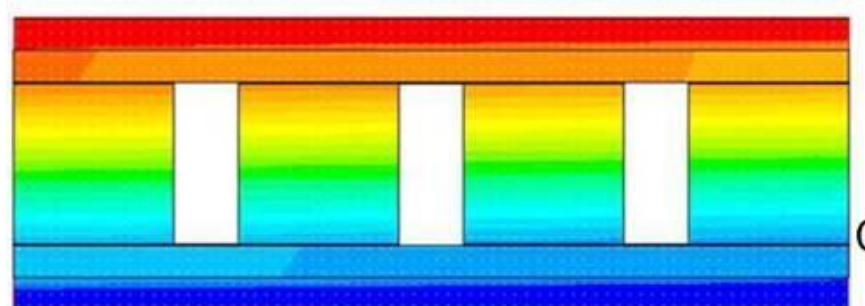

Contours of Temp.(K)

Fig.1 Temperature distributions of simple counter flow when the fluid velocity is $0.001 \mathrm{~m} / \mathrm{s}(\mathrm{a}), 0.005 \mathrm{~m} / \mathrm{s}(\mathrm{b}), 0.01 \mathrm{~m} / \mathrm{s}(\mathrm{c})$ and $0.05 \mathrm{~m} / \mathrm{s}(\mathrm{d})$. 

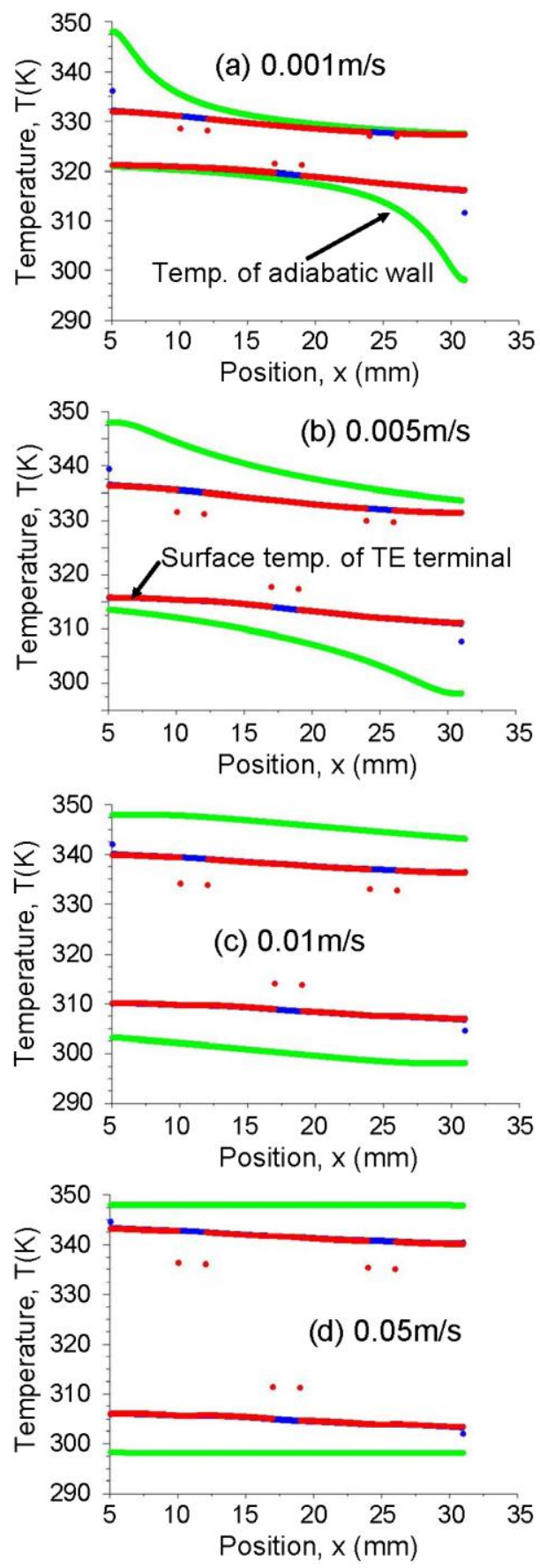

Fig.2 Temperature distribution of simple counter flow along the flow direction. 


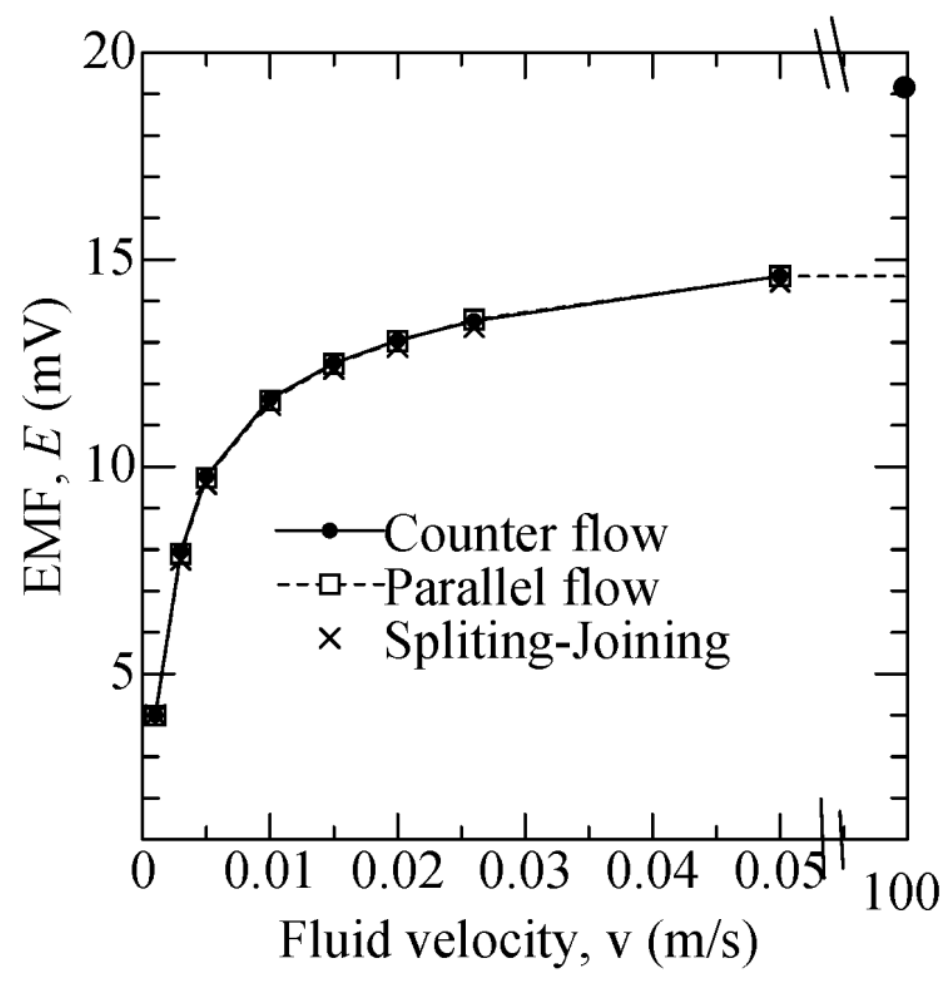

Fig.3 Relationship between Seebeck EMF and fluid velocity of simple counter flow. 


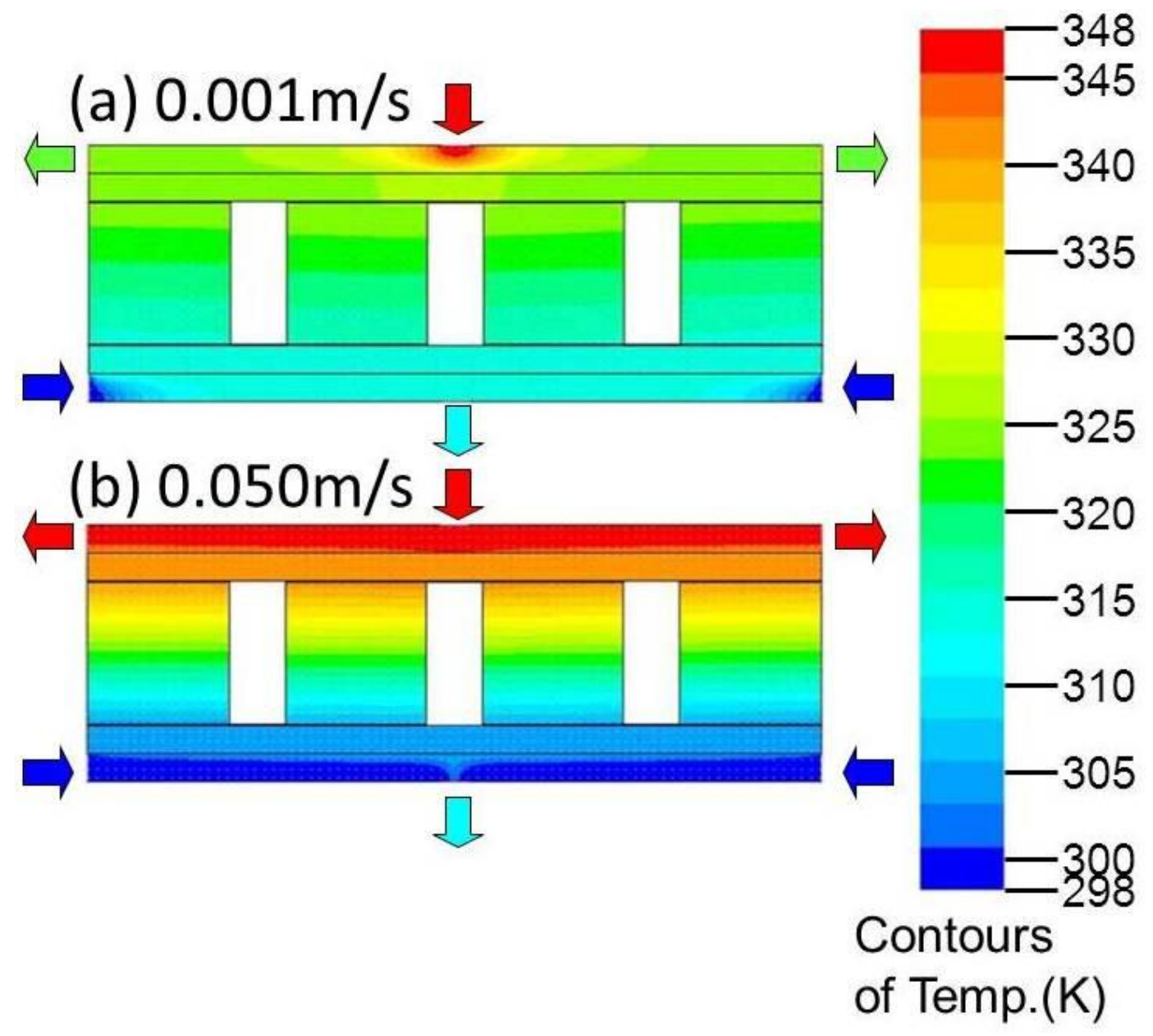

Fig.4 Temperature distributions of splitting and joining flow when the fluid velocity is $0.001 \mathrm{~m} / \mathrm{s}(\mathrm{a})$ and $0.05 \mathrm{~m} / \mathrm{s}(\mathrm{b})$. 

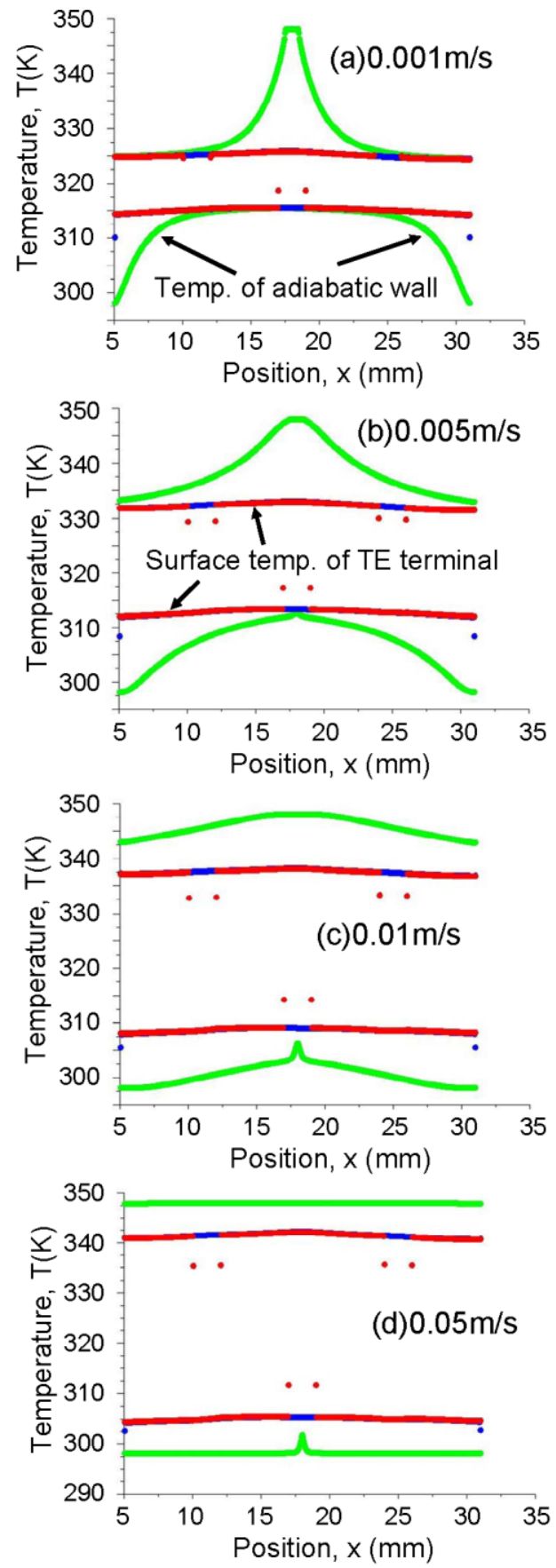

Fig.5 Temperature distribution of splitting and joining flow along the flow direction. 
(a)

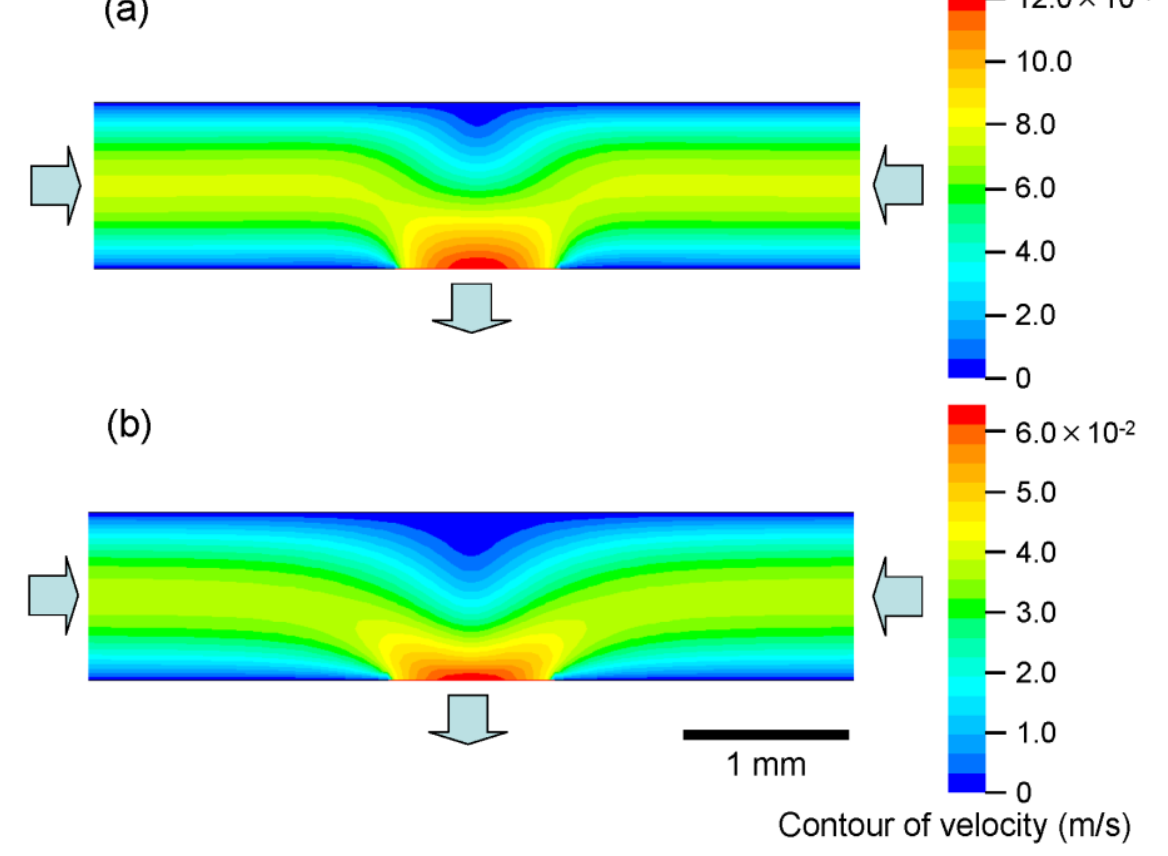

Fig.6 Velocity of cold fluid when "joining” occurs at the center. 